\title{
Foliated fields of an extension of a Lie G-foliation having dense leaves on a compact manifold.
}

\author{
Cyrille Dadi $^{(1)}$ and Adolphe Codjia ${ }^{(2)}$ \\ Fundamental Mathematics Laboratory, \\ University Felix Houphouet-Boigny, ENS \\ 08 PO Box 10 Abidjan ( Ivory Coast) \\ email $^{(1)}$ :cyriledadi@yahoo.fr,email( ${ }^{(2)}:$ ad_wolf2000@yahoo.fr
}

March 10, 2018

\begin{abstract}
Is shown in ([3], [4]) as any extension of a $\mathcal{G}$-foliation $\mathcal{F}$ having dense leaves on a compact manifold $M$ corresponds a Lie subalgebra $\mathcal{H}$ of $\mathcal{G}$.

We denote by $\mathcal{F}_{\mathcal{H}}$ the extension corresponding to the subalgebra $\mathcal{H}$.

In this paper we seek to determine the Lie algebra $\ell\left(M, \mathcal{F}_{\mathcal{H}}\right)$ of transverse foliated fields of the extension $\mathcal{F}_{\mathcal{H}}$.

This study show us that

$$
\ell\left(M, \mathcal{F}_{\mathcal{H}}\right)=\left\{u \in \mathcal{H}^{\perp} /[u, h]=0 \text { for every } h \in \mathcal{H}\right\} .
$$

Key words and phrases: Lie foliation, Riemannian foliation, foliation having dense leaves, extension of a foliation .

Subject Classifications: 53C05, 53C12, 58A30.

\section{Introduction}

Is shown in $([3],[4])$ as any extension of $\mathcal{G}$-foliation $\mathcal{F}$ having dense leaves on a compact manifold $M$ corresponds a Lie subalgebra $\mathcal{H}$ of $\mathcal{G}$.

We denote by $\mathcal{F}_{\mathcal{H}}$ the extension corresponding to the subalgebra $\mathcal{H}$.

The purpose of this article is to compute the Lie algebra $\ell\left(M, \mathcal{F}_{\mathcal{H}}\right)$ of $\mathcal{F}_{\mathcal{H}^{-}}$ foliated transverse vectors fields.

The following results were first established:

(i) all extension $\mathcal{F}^{\prime}$ of a Riemannian foliation $\mathcal{F}$ having dense leaves on a compact manifold is a Riemannian foliation
\end{abstract}


(ii) If $\mathcal{F}^{\prime \prime}$ is an extension of Riemannian foliation $\mathcal{F}^{\prime}$ on a compact manifold $M$ then $\ell\left(M, \mathcal{F}^{\prime \prime}\right) \subset \ell\left(M, \mathcal{F}^{\prime}\right)$.

That said, we show in this paper that all informations about $\ell\left(M, \mathcal{F}_{\mathcal{H}}\right)$ are contained in the Lie algebra $\mathcal{G}$ of $G$.

In fact, it is established to an isomorphism of Lie algebra nearly that:

$$
\ell\left(M, \mathcal{F}_{\mathcal{H}}\right)=\left\{u \in \mathcal{H}^{\perp} /[u, h]=0 \text { for all } h \in \mathcal{H}\right\}
$$

This paper is articulated in three parts:

- The first part is devoted to reminders about extensions of foliation and $\frac{\mathcal{G}}{\mathcal{H}}$-foliations,

- Extension of Riemannian foliation having dense leaves on a compact manifold,

- The third part is devoted to computation of $\ell\left(M, \mathcal{F}_{\mathcal{H}}\right)$.

\section{Definitions and Reminders}

In this section, are formulated in the helpful sense some definitions and theorems which are in $([3],[4],[5])$.

Definition 2.1 An extension of a codimension $q$ foliation $(M, \mathcal{F})$ is a codimension $q^{\prime}$ foliation $\left(M, \mathcal{F}^{\prime}\right)$ such as $0<q^{\prime}<q$ and the leaves of $\left(M, \mathcal{F}^{\prime}\right)$ are union of those of $(M, \mathcal{F})$ (we note $\left.\mathcal{F} \subset \mathcal{F}^{\prime}\right)$.

We show that if $\left(M, \mathcal{F}^{\prime}\right)$ is a simple extension of a simple foliation $(M, \mathcal{F})$ and if $(M, \mathcal{F})$ and $\left(M, \mathcal{F}^{\prime}\right)$ are respectively defined by submersions $\pi: M \rightarrow T$ and $\pi^{\prime}: M \rightarrow T^{\prime}$, then there exists a submersion $\theta: T \rightarrow T^{\prime}$ such as $\pi^{\prime}=\theta \circ \pi$.

We say that the submersion $\theta$ is a bond between the foliation $(M, \mathcal{F})$ and its extension $\left(M, \mathcal{F}^{\prime}\right)$.

It is shown in [3] if the foliation $(M, \mathcal{F})$ and its extension $\left(M, \mathcal{F}^{\prime}\right)$ are respectively defined by the cocycles $\left(U_{i}, f_{i}, T, \gamma_{i j}\right)_{i \in I}$ and $\left(U_{i}, f_{i}^{\prime}, T^{\prime}, \gamma_{i j}^{\prime}\right)_{i \in I}$ then we have:

$$
f_{i}^{\prime}=\theta_{i} \circ f_{i} \text { and } \gamma_{i j}^{\prime} \circ \theta_{j}=\theta_{i} \circ \gamma_{i j}
$$

where $\theta_{s}$ is a bond between the foliation $\left(U_{s}, \mathcal{F}\right)$ and its extension $\left(U_{s}, \mathcal{F}^{\prime}\right)$.

In what follows $\mathcal{G}$ is the Lie algebra of a Lie group $G$ of dimension $q, \mathcal{H}$ is a Lie

subalgebra of $\mathcal{G}$ of codimension $q^{\prime},\left(e_{1}, \ldots, e_{q}\right)$ is a base of $\mathcal{G}$ such as $\left(e_{q_{+1}^{\prime}}, \ldots, e_{q}\right)$ may be a base of $\mathcal{H}, \omega$ be a 1 -form on a manifold $M$ taking values in $\mathcal{G}$, relatively to $\left(e_{1}, \ldots, e_{q}\right)$ we have $\omega=\sum_{i=1}^{q} \omega^{i} \otimes e_{i}$. What is written $\omega=\left(\omega^{1}, \ldots, \omega^{q}\right)$.

If $d \omega+\frac{1}{2}[\omega, \omega]=0$ and $\omega^{1}, \ldots, \omega^{q^{\prime}}$ are linearly independent at any point of $M$ then the differential system $\omega^{1}=\ldots=\omega^{q^{\prime}}=0$ define a foliation $\mathcal{F}_{\mathcal{H}}$ of codimension $q^{\prime}$.

Definition 2.2 The foliation $\mathcal{F}_{\mathcal{H}}$ defined is called $\frac{\mathcal{G}}{\mathcal{H}}$-foliation defined by the 1-form $\omega$. 
Proposition 2.3 Let $\mathcal{F}_{\mathcal{H}}$ be a $\frac{\mathcal{G}}{\mathcal{H}}$-foliation defined by a 1-form $\omega$ and let $\widetilde{\mathcal{F}}_{\mathcal{H}}=$ $\pi^{*} \mathcal{F}_{\mathcal{H}}$ be the lifting of the $\mathcal{F}_{\mathcal{H}}$-foliation on the universal covering $\pi: \widetilde{M} \rightarrow M$ of $M$.

Then, there is a differentiable map $\mathcal{D}: \widetilde{M} \rightarrow G$ transverse for the foliation $\mathcal{F}_{G, H}$ obtained by the left translations of $H$ that is to say that for every $\widetilde{x} \in \widetilde{M}$,

$$
T_{\mathcal{D}(\widetilde{x})} G=T_{\widetilde{x}} \mathcal{D}\left(T_{\widetilde{x}} \widetilde{M}\right)+T_{\mathcal{D}(\widetilde{x})} \mathcal{F}_{G, H}
$$

and a homomorphism $\rho: \pi_{1}(M) \rightarrow G$ such as:

(i) $\mathcal{D}$ is $\rho$-equivariant that is to say that

$$
\mathcal{D}(\gamma \cdot \widetilde{x})=\rho(\gamma) \cdot \mathcal{D}(\widetilde{x}),
$$

$$
\pi^{*} \omega=\mathcal{D}^{*} \alpha \text { that is to say } \widetilde{\mathcal{F}}_{\mathcal{H}}=\mathcal{D}^{*} \mathcal{F}_{G, H}
$$

where $\alpha$ is the Maurer-Cartan form over $G$.

It is saying that $\mathcal{D}$ is an involute application on $\widetilde{M}$ of the $\frac{\mathcal{G}}{\mathcal{H}}$-foliation $\mathcal{F}_{\mathcal{H}}$.

If the 1-form $\omega$ is Fedida's 1-form of a Lie-foliation $\mathcal{F}$, then all involute application of $\mathcal{F}$ is also involute application of $\mathcal{F}_{\mathcal{H}}$.

Before finishing this part of recall, we give the theorem of the biunivocal correspondence between Lie subalgebras of $\mathcal{G}=\operatorname{Lie}(G)$ and the extensions of a Lie $G$-foliation having dense leaves existing in [4].

Theorem 2.4 Let $(M, \mathcal{F})$ be a Lie $G$-foliation having dense leaves on a compact, connected manifold, with Lie algebra $\mathcal{G}$.

Then:

1-There is a correspondence 1-to-1 between Lie-subalgebra of $\mathcal{G}=$ Lie $(G)$ (or if you prefer between the conncted Lie subgroup of $G$ ) and the extensions of $\mathcal{F}$.

2- An extension of $\mathcal{F}$ is a $\frac{\mathcal{G}}{\mathcal{H}}$ transverse riemannian foliation with trivial normal fiber bundle, defined by 1-forme valued in $\frac{\mathcal{G}}{\mathcal{H}}$.

3- An extension of $\mathcal{F}$ is transversally homogeneous (resp. of Lie) if and only if the Lie subgroup of $G$ corresponding to it is a closed subgroup(resp. a normal subgroup) of $G$.

Remark 2.5 Let $\mathcal{F}_{\mathcal{H}}$ be an extension of a Lie $G$-foliation with dense leaves on a compact manifold corresponding to the Lie-subalgebra $\mathcal{H}$ of $\mathcal{G}=\operatorname{Lie}(G)$, let $\pi: \widetilde{M} \rightarrow M$ be the universal covering of $M$, let $\mathcal{D}: \widetilde{M} \rightarrow G$ be the involute application of $\mathcal{F}$ associated to a 1-form of Fedida $\omega$, let $\alpha$ be the Maurer-Cartan form on $G$, let $\widetilde{\mathcal{F}}_{\mathcal{H}}$ be the lift of $\mathcal{F}_{\mathcal{H}}$ on $\widetilde{M}$, let $U$ be a distinguished open set in $\widetilde{M}$ for both $\mathcal{F}$ and $\mathcal{F}_{\mathcal{H}}$, let $f^{\prime}$ be a submersion defining $\mathcal{F}_{\mathcal{H}}$ on $U$ and let $\overline{\bar{U}}$ be a local quotient manifold of $\mathcal{F}_{\mathcal{H}}$ on $U$.

i) There is an open set $\widetilde{U}$ of $\widetilde{M}$, an open set $\bar{U}$ of $G$, a riemannian submersion $f: U \rightarrow \bar{U}$ and a riemannian submersion $\theta: \bar{U} \rightarrow \overline{\bar{U}}$ such that following diagram 
commutes:

$$
\begin{array}{ccc}
\widetilde{U} & \stackrel{\mathcal{D}}{\rightarrow} & \bar{U} \\
\pi \downarrow & \nearrow_{f}^{f} & \downarrow \theta . \\
U & \stackrel{f^{\prime}}{\rightarrow} & \overline{\bar{U}}
\end{array}
$$

We have $f: U \rightarrow \bar{U}$ defining $\mathcal{F}$ on $U$ and $\theta$ is a bond between $(U, \mathcal{F})$ and $\left(U, \mathcal{F}_{\mathcal{H}}\right)$.

ii) The Darboux's differential of $f$ is $\omega$ that is to say that

$$
\omega_{x}\left(X_{x}\right)=\left(L_{f(x) *}\right)^{-1} \circ f_{* x}\left(X_{x}\right)
$$

for every $X_{x} \in T_{x} U$.

iii) There is

$$
f_{* x}\left(T_{x} \mathcal{F}_{\mathcal{H}}\right)=L_{f(x) *}(\mathcal{H})=T_{x} \mathcal{F}_{\theta}
$$

for every $x \in U$ that is to say that

$$
f\left(\mathcal{F}_{\mathcal{H}}\right)=\mathcal{F}_{\overline{U, H}}=\mathcal{F}_{\theta}
$$

where $\mathcal{F}_{\bar{U}, H}$ is the restriction to $\bar{U}$ of $\mathcal{F}_{G, H}$.

iv) For $X_{x} \in T_{x} M$,

$$
X_{x} \in T_{x} \mathcal{F}_{\mathcal{H}} \Longleftrightarrow \omega_{x}\left(X_{x}\right) \in \mathcal{H} .
$$

v) $\mathcal{F}$ being with dense leaves, we note that for every vector fields $X$ and $Y$, $\mathcal{F}$-transverse foliated leads

$$
\omega([X, Y])=[\omega(X), \omega(Y)] .
$$

vi) Let $\mathcal{X}\left(\mathcal{F}_{\mathcal{H}}\right)$ (resp. $\mathcal{X}(\mathcal{F})$ ) be the Lie algebra of tangent vectors fields of $\mathcal{F}_{\mathcal{H}}$ (resp. $\left.\mathcal{F}\right)$, let $\mathcal{A}^{1}(M)$ be the space of differentiable fonctions on $M$ and let $\widetilde{\mathcal{H}}$ be the Lie algebra of vectors fields $\mathcal{F}$-foliated obtained from $\mathcal{H}$.

We have ([3], [4])

$$
\mathcal{X}\left(\mathcal{F}_{\mathcal{H}}\right)=\mathcal{X}(\mathcal{F}) \oplus\left(\mathcal{A}^{1}(M) \otimes \widetilde{\mathcal{H}}\right) .
$$

We end this part with the two following propositions found in [9] and which will be useful in the following.

Proposition 2.6 $[9]$ Let $\mathcal{K}(M, \lambda)$ be a Lie algebra of Killing vectors fields on a Riemannian connected manifold $(M, \lambda)$, let $i_{x}=\left\{X \in \mathcal{K}(M, \lambda) / X_{x}=0\right\}$ be the isotropy of $\mathcal{K}(M, \lambda)$ at the point $x \in(M, \lambda)$, let $\mathcal{O}_{x}$ be the orbit at the point $x$ of $\mathcal{K}(M, \lambda)$ and let $\mathcal{O}_{\left(x_{0}, x\right)}$ be the orbit at the point $x \in(M, \lambda)$ of the isotropy $i_{x_{0}}$ where $x_{0} \in(M, \lambda)$.

If for $x \in(M, \lambda)$, all orbits $\mathcal{O}_{x}$ have the same dimension then $\mathcal{O}_{\left(x_{0}, x\right)}=\{x\}$ for every $\left(x_{0}, x\right) \in(M, \lambda)^{2}$ 
This proposition implies that if $X$ and $Y$ are two Killing vectors fields on a related open set $U$ and $a \in U$ then

$$
X_{a}=Y_{a} \Longleftrightarrow X=Y .
$$

Proposition 2.7 ([?], [9]) Let $\mathcal{F}$ be a codimension $q$ Riemannian foliation on a compact connected manifold $M$, let $\overline{F^{\natural}}$ be the closure of a leaf $F^{\natural}$ of lifted foliation $\mathcal{F}^{\natural}$ of $\mathcal{F}$ on the orthonormal transverse frame bundle $M^{\natural}$, let $\phi: M^{\natural} \rightarrow M$ be the projection which to a frame at $x$ associates $x$.

Them:

i) $\phi\left(F^{\natural}\right)$ is a leaf of $\mathcal{F}$ and $\phi\left(\overline{F^{\natural}}\right)=\overline{\phi\left(F^{\natural}\right)}$.

ii) the map $\phi: \overline{F^{\natural}} \rightarrow \phi\left(\overline{F^{\natural}}\right)$ is a locally trivial fibration .

iii) $\phi: \overline{F^{\natural}} \rightarrow M$ is a covering.

\section{Extension of Riemannian foliation having dense leaves on a compact manifold}

In the following we designate by $\widetilde{k}$ the transverse vector field associated with $k \in \mathcal{G}$ where $\mathcal{G}=\operatorname{Lie}(G)$.

Proposition 3.1 Let $(M, \lambda)$ be a Riemannian manifold, let $\widetilde{\mathcal{H}}$ be a Lie algebra of Killing vector fields on the Riemannian manifold $(M, \lambda)$ and let $\mathcal{H}_{M}^{\perp}$ be a set of all vector fields orthogonal to any vector fields of $\mathcal{H}$.

Then for every $\widetilde{h} \in \widetilde{\mathcal{H}}$ and every $\widetilde{u} \in \mathcal{H}_{M}^{\perp}$ we have

$$
[\widetilde{h}, \widetilde{u}] \in \mathcal{H}_{M}^{\perp}
$$

Proof. Let $\widetilde{k} \in \widetilde{\mathcal{H}}$ be a voctor field, Let $\left(\widetilde{h}, \widetilde{h^{\prime}}\right) \in \widetilde{\mathcal{H}} \times \widetilde{\mathcal{H}}$ and let $\widetilde{u} \in \mathcal{H}_{M}^{\perp}$ we have

$$
\begin{aligned}
0 & =\widetilde{h^{\prime}} \lambda(\widetilde{u}, \widetilde{h}) \\
& =\lambda\left(\left[\widetilde{h^{\prime}}, \widetilde{u}\right], \widetilde{h}\right)+\lambda\left(\widetilde{u},\left[\widetilde{h^{\prime}}, \widetilde{h}\right]\right) \text { because } \widetilde{h^{\prime}} \text { is a Killing vector field for } \lambda \\
& =\lambda\left(\left[\widetilde{h^{\prime}}, \widetilde{u}\right], \widetilde{h}\right)+0 \\
& =\lambda\left(\left[\widetilde{h^{\prime}}, \widetilde{u}\right], \widetilde{h}\right)
\end{aligned}
$$

The equality $\lambda\left(\left[\widetilde{h^{\prime}}, \widetilde{u}\right], \widetilde{h}\right)=0$ for all $\left(\widetilde{h^{\prime}}, \widetilde{u}\right) \in \widetilde{\mathcal{H}} \times \mathcal{H}_{M}^{\perp}$ implies that $\left[\widetilde{h^{\prime}}, \widetilde{u}\right] \in$ $\mathcal{H}_{M}^{\perp}$ for all $\left(\widetilde{h^{\prime}}, \widetilde{u}\right) \in \widetilde{\mathcal{H}} \times \mathcal{H}_{M}^{\perp}$. 
Proposition 3.2 [7] Let $(M, \mathcal{F})$ be a transversaly oriental Riemannian foliation on a manifold compact connected manifolde .

The adhesions of the leaves of $(M, \mathcal{F})$ correspond to a differential system $\mathcal{P}$ on $M$ such as $\frac{\mathcal{P}}{T \mathcal{F}}$ can be generated locally by transversal Killing vectors fields $Z_{1}, \ldots, Z_{r}$ for $(M, \mathcal{F})$ having the following properties:

i) $Z_{1}, \ldots, Z_{r}$ switch with transverse foliated fields.

ii) $Z_{1}, \ldots, Z_{r}$ freely generate a Lie algebra isomorphic to structural Lie algebra $\mathcal{G}$ of $(M, \mathcal{F})$.

The previous proposition implies that for any global foliated transverse vector field $\bar{X}$ and $a \in M$, there existe an open set $\mathcal{F}$-distinguished $U$ having $\bar{U}$ for $\mathcal{F}$ - local quotient containing $\bar{a}$ such as $\bar{X}_{\bar{U}}$ is a Killing vector field $s$ where $\bar{a}$ is the project of $a$ following $\mathcal{F}$. Indeed consider the previous $\mathcal{F}$-distinguished set $U$, let $\bar{U}$ be the $\mathcal{F}$-quotient local of $U$ on where the Killing $\mathcal{F}$-transverse vectors fields $Z_{1}, \ldots, Z_{r}$ switching with globaltransverse foliated fields are defined where $r=\operatorname{codim} \mathcal{F}(c f \cdot$ proposition $[7]) \cdot$ Let $Z_{k} \in \mathcal{G}$ and let $\mathcal{F}_{Z_{k}}$ (resp. $\left.\mathcal{F}_{X}\right)$ be foliations on $\bar{U}$ definied by the vector field $Z_{k}$ (resp. $X$ ), we assume that the open set $\bar{U}$ is both distinguished for $\mathcal{F}_{Z_{k}}$ and $\mathcal{F}_{X}$.

In all that follows it will be assumed that $U$ will be chosen so that $\bar{U}$ is a connected set · Let $\bar{U}$ be the $\mathcal{F}_{Z_{k}}-$ local quotient of $\bar{U}$ and let $\bar{a} \in U$. For all vector $\bar{X}_{\bar{a}}$ at a point $\bar{a}$ there existe a $\mathcal{F}$-transverse Killing vectors field $Z_{r}$ such as

$$
Z_{r}(\bar{a})=\bar{X}_{\bar{a}}
$$

We have

$$
\left(Z_{r}-\bar{X}\right)(\bar{a})=0
$$

As

$$
\left[Z_{r}-\bar{X}, Z_{j}\right]=0 \text { for all } j \in\{1, \ldots, r\}
$$

and the transverse vectors fields $Z_{1}, \ldots, Z_{r}$ form a parallelism of $\bar{U}$ then

$$
\Omega=\{\bar{x} \in \bar{U} / Y(\bar{x})=0\}
$$

where

$$
Y=Z_{r}-\bar{X}
$$

is an open set [7] closed not empty. Therefore the fact $\bar{U}$ is a connected set implies that $\Omega=\bar{U}$.

Thus, its established that $\bar{X}_{/ \bar{U}}$ is a Killinvector fields.

Corollary 3.3 Let $\mathcal{H}$ be a Lie subalgebra of the Lie algebra $\mathcal{G}$ of a Lie foliation $\mathcal{F}$ having dense leaves

on a compact manifold and $u \in \mathcal{H}^{\perp}$.

then the following assertions are equivalent:

i) $[u, h]=0$ for every $h \in \mathcal{H}$.

ii) $[u, h] \in \mathcal{H}$ for every $h \in \mathcal{H}$ 
Proof. According the proposions $(3 \cdot 1)$ and $(3 \cdot 2),[u, h] \in \mathcal{H}^{\perp}$. Therefore, the fact that

$$
[u, h] \in \mathcal{H} \Longleftrightarrow[u, h]=0 .
$$

In what follows we consider the proposal $3 \cdot 2$.

We designate by $U$ an open set on which the transverses Killing vector $Z_{1}, \ldots, Z_{r}$ are defined.

We will say that the adhesions of the leaves of $(M, \mathcal{F})$ are the orbits $[1,1]$ of the transverse beam $\mathcal{C}_{U}(\mathcal{F}, \mathcal{G})$ of $\mathcal{F}$ having $\mathcal{G}$ for fiber type .

In the following we will show that any extension of a Riemannian foliation having dense leaves have its leaves which are orbits of a transverse beam.

Theorem 3.4 Let $\mathcal{F}$ be a Riemannian foliation having dense leaves on on a Riemannian compact manifold $(M, \lambda) \cdot$ Let $M^{\natural}$ be the fiber of the transverse orthonormal frame bundle of $\mathcal{F}$, let $\mathcal{F}$ घbe the lifted foliation of $\mathcal{F}$ on $M^{\natural}$, let $F^{\natural}$ be a leaf of $\mathcal{F}^{\natural}$, let $\overline{F^{\natural}}$ be the closure of $F^{\natural}$, let $\phi: M^{\natural} \rightarrow M$ be the projection to a frame at $x$ associated $x$, let $\mathcal{G}$ be the Lie structural algebral of $\mathcal{F}$ and let $\widetilde{\mathcal{F}}$ be an extension of $\mathcal{F}$.

Then :

(i) The leaves of foliation $\widetilde{\mathcal{F}}^{\natural}=\phi^{*}(\widetilde{\mathcal{F}})=\phi^{-1}(\widetilde{\mathcal{F}})$ are the orbits $([1,1])$ of a transverse beam $\mathcal{C}_{U}(\widetilde{\mathcal{F}}, \mathcal{H})$ of $\widetilde{\mathcal{F}}$ having $\mathcal{H} \subset \mathcal{G}$ for fiber ype.

(ii) $\widetilde{\mathcal{F}}$ is a riemannian foliation.

Proof. (i) Let $\tilde{\lambda}=\phi^{*}(\lambda)$ the metric pulls back of $\lambda$ and let $\mathcal{V}_{x}(\mathcal{F})=$ $\left(T_{x} \mathcal{F}\right)^{\perp}$ be the fiber at $x$ of orthonormal fiber $\mathcal{V}(\mathcal{F}) \cdot \tilde{\lambda}$ is a metrique on $\overline{F^{\natural}}$ because $\phi: \overline{F^{\natural}} \rightarrow M$ is a covering [9].

Note that the restriction $\mathcal{F}_{\overline{F^{\natural}}}^{\natural}$ of $\widetilde{\mathcal{F}}^{\natural}$ on $\overline{F^{\natural}}$ is a Lie $\mathcal{G}$ foliation having dense leaves [7], We also note that the foliaton $\widetilde{\mathcal{F}}^{\natural}=\phi^{*}(\widetilde{\mathcal{F}})$ is by construction an extension of the Lie foliation having dense leaves $\mathcal{F}_{\overline{F^{\natural}}}^{\natural}$ because $\mathcal{F} \subset \widetilde{\mathcal{F}}$ and $\widetilde{\mathcal{F}}^{\natural}=\phi^{*}(\widetilde{\mathcal{F}})$ and $\mathcal{F}^{\natural}\left(\operatorname{resp} \cdot \widetilde{\mathcal{F}}^{\natural}\right)$ are the lifted foliation of $\mathcal{F}($ resp $\cdot \widetilde{\mathcal{F}})$ on $M^{\natural}$. Acording to the theoreme $2 \cdot 4, \widetilde{\mathcal{F}}^{\natural}$ is a Riemannian foliation having dense leaves on the Riemannian compact manifold $(M, \lambda)$ then there existe $\mathrm{r}$ local Killing vecteurs fields $Z_{1}^{\natural} \ldots, Z_{r}^{\natural}$ linearly independent such as according to the propositions $([7],[9])$

$$
\left(T_{x^{\natural}} \widetilde{\mathcal{F}}^{\natural}\right)^{\perp}=e v_{x^{\natural}}(\mathcal{G})=\left\langle Z_{1}^{\natural} \ldots, Z_{r}^{\natural}\right\rangle
$$


where $e v_{x}(X)=X_{x}$. And according the proposition [9] $\phi: M^{\natural} \rightarrow M$ is a covering, Then

$$
\begin{aligned}
\left(T_{x} \widetilde{\mathcal{F}}\right)^{\perp} & =\phi_{*}\left(\left(T_{x^{\natural}} \widetilde{\mathcal{F}}^{\natural}\right)^{\perp}\right) \text { because relatively to } \lambda \text { and } \widetilde{\lambda}, \phi \text { is a local isometry and } \phi\left(x^{\natural}\right)=x \\
& =\left\langle\phi_{*}\left(Z_{1 x^{\natural}}^{\natural}\right),\left(\phi_{*}\left(Z_{2 x^{\natural}}^{\natural}\right)\right), \ldots, \phi_{*}\left(Z_{r x^{\natural}}^{\natural}\right)\right\rangle \\
& \simeq e v_{x^{\natural}}(\mathcal{G}) \\
& \simeq \mathcal{G}
\end{aligned}
$$

From the foregoing it is argued that the leaves of $\widetilde{\mathcal{F}}$ are the orbits ([1]) of transverse beam $\mathcal{C}_{U}(\widetilde{\mathcal{F}}, \mathcal{G})$ of $\widetilde{\mathcal{F}}$ having $\overline{\mathcal{G}}=(\phi)_{*}(\mathcal{G}) \backsim \mathcal{G}$ for fiber type.

(ii) what precedes shows that $\widetilde{\mathcal{F}}$ is a riemannian foliation. In deed

let's show now that foliation $\widetilde{\mathcal{F}}$ is Riemannian. For it, as the problem is local, notice that:

an extension $\widetilde{\mathcal{F}}$ of a Riemannian foliation $\mathcal{F}$ is a Riemannian foliation

if and only if there existe an open set $U$ such $(U, \mathcal{F})$ is a Riemannian foliation.

As $\phi: M^{\natural} \rightarrow M$ is a covering, then there existe an open set $U^{\natural}$ such as:

(i) $\phi: U^{\natural} \longrightarrow U$ is a is a difeomorphism

(ii) $\left(U^{\natural}, \widetilde{\mathcal{F}}^{\natural}\right)$ and $(U, \widetilde{\mathcal{F}})$ are simple.

Note that $(U, \mathcal{F})$ and $\left(U^{\natural}, \widetilde{\mathcal{F}}^{\natural}\right)$ are difeormorphic then $(U, \mathcal{F})$ is Riemannian if and only if $\left(U^{\natural}, \widetilde{\mathcal{F}}^{\natural}\right)$ is Riemannian. Therefore the fact that $\widetilde{\mathcal{F}}^{\natural}$ is a Riemannian foliation implies that $(U, \widetilde{\mathcal{F}})$ is a Riemannian foliation. Thus all extension $\widetilde{\mathcal{F}}$ of a Riemannian foliation $\mathcal{F}$ having dense leaves is a Riemannian foliation.

\section{Computation of the Lie algebra of the set of foliated transverse vectors fields of an exten- sion of a Lie $\mathrm{G}$ foliation having dense leaves on a compact manifold}

Proposition 4.1 Let $\mathcal{G}$ be the Lie structural algebra of a Lie G foliation having dense leaves, let $\mathcal{H}$ be a Lie subalgebra of $\mathcal{G}=$ Lie $(G)$, and let $\mathcal{H}^{\perp}$ be the orthocomplentary of $\mathcal{H}$ in $\mathcal{G}$ relavely to $\lambda$.

Then

$$
\left\{u \in \mathcal{H}^{\perp} /[u, h]=0 \quad \text { for every } h \in \mathcal{H}\right\}
$$

is a Lie subagebra of $\mathcal{G}$. 
Proof. We consider two vectors $u$ and $v$ of $\mathcal{B}=\left\{u \in \mathcal{H}^{\perp} /[u, h]=0\right.$ for every $h \in \mathcal{H}\} \cdot$ Note Above all that

$$
[t, z]=0 \text { for all } t \in \mathcal{B} \quad \text { and } z \in \mathcal{H}
$$

On other hand for $k \in \mathcal{H}$ we have

$0=[[u, v], k]+[[v, k], u]+[[k, u], v]=[[u, v], k]+[0, u]+[0, v]=[[u, v], k]$

morever, according to Molino there exists an open neighborhood $V_{e}$ of the neutral element $e$ of $G$ such as for all $b \in \mathcal{G}$ the restrition $\widetilde{b}_{V_{e}}$ of the transverse vector fields associted at $b \in \mathcal{G}$ is a Killing vector. From where, we have

$$
\begin{aligned}
0 & =\widetilde{u}_{V_{e}} \cdot \lambda\left(\widetilde{v}_{V_{e}}, \widetilde{k}_{V_{e}}\right) \text { because } \lambda\left(\widetilde{v}_{V_{e}}, \widetilde{k}_{V_{e}}\right)=\lambda(v, k) \\
& =\lambda\left(\left[\widetilde{u}_{V_{e}}, \widetilde{v}_{V_{e}}\right], \widetilde{k}_{V_{e}}\right)+\lambda\left(\widetilde{v}_{V_{e}},\left[\widetilde{u}_{V_{e}}, \widetilde{k}_{V_{e}}\right]\right) \\
& =\lambda\left(\left[\widetilde{u}_{V_{e}}, \widetilde{v}_{V_{e}}\right], \widetilde{k}_{V_{e}}\right)+\widetilde{k}_{V_{e}}\left(\widetilde{v}_{V_{e}}, 0\right) \\
& =\lambda\left(\left[\widetilde{u}_{V_{e}}, \widetilde{v}_{V_{e}}\right], \widetilde{k}_{V_{e}}\right) \\
& =\lambda\left(\widetilde{[u, v]}_{V_{e}}, \widetilde{k}_{V_{e}}\right) \\
& =\lambda([[u, v], k]) \cdot \quad(\star \star \star) \quad \text { Thus }[u, v] \in \mathcal{H}^{\perp} .
\end{aligned}
$$

The egality $(\star \star \star)$ and $(\star \star)$ implies that .

$$
\left\{u \in \mathcal{H}^{\perp} /[u, h]=0 \text { for every } h \in \mathcal{H}\right\}
$$

is a Lie subagebra of

Let $\mathcal{G}$ be the Lie algebra of a Lie group $G$ and let $h_{T}$ be a left-invariant metric on $G$.

Let $\mathcal{F}_{\mathcal{H}}$ the extension of Lie $G$-foliation having dense leaves $\mathcal{F}$ on a compact, connected

manifold corresponding to subalgebra $\mathcal{H}$ of $\mathcal{G}=\operatorname{Lie}(G), \mathcal{H}^{g}$ The Lie

algebra of left-invariant vector fields on $G$ obtained from vectors of Lie subalgebra $\mathcal{H}$ of $\mathcal{G}$ and $\mathcal{F}_{G, H}$ the foliation on $G$ whose leaves are left classes of $H$.

The above theorem allows us to say that the leaves of the foliation $\mathcal{F}_{G, H}$ ( resp. $\mathcal{F}_{\mathcal{H}}$ ) are orbits (resp. transversally to $\mathcal{F}$ ) of a Lie algebra of Killing fields. And this Lie algebra is of course $\mathcal{H}^{g}$.

In the following if there is a metric $h$ on a manifold $M$, we will identify for every

foliation $\mathcal{F}^{\prime}$ on $M$, the normal bundle $\mathcal{V}\left(\mathcal{F}^{\prime}\right)$ of $\mathcal{F}^{\prime}$ and the orthogonal bundle $\left(T \mathcal{F}^{\prime}\right)^{\perp}$ of $T \mathcal{F}^{\prime}$.

The purpose of this section is to calculate the algebra $\ell\left(M, \mathcal{F}_{\mathcal{H}}\right)$ of vector

fields $\mathcal{F}_{\mathcal{H}}$-transverse foliate where $\mathcal{F}_{\mathcal{H}}$ is an extension of Lie $G$-foliation having dense 
leaves $\mathcal{F}$ on compact, connected manifold corresponding to the subalgebra $\mathcal{H}$ of $\mathcal{G}=\operatorname{Lie}(G)$.

Before stating the next proposal, we recall some remarks on the transversally parallelizable foliations on a compact manifold.

Let $\mathcal{F}^{\prime}$ a transversally parallelizable foliation on a compact manifold $M$, $\mathcal{F}_{b}^{\prime}$ the basic foliation of $\mathcal{F}^{\prime}$ and $\pi: M \rightarrow W$ the basic fibration of $\mathcal{F}^{\prime}$. It is known [7] that $\pi$ defined $\mathcal{F}_{b}^{\prime}$ and we have the following exact sequence:

$$
0 \rightarrow \underline{\varepsilon}_{w} \rightarrow \ell\left(M, \mathcal{F}^{\prime}\right) \rightarrow \mathcal{X}(W) \rightarrow 0
$$

where $\underline{\varepsilon}_{w}$ is the Lie algebra of vector fields $\mathcal{F}^{\prime}$-transverse foliate which projection by $\pi$ is zero and $\mathcal{X}(W)$ is the Lie algebra of vector fields of the basic manifold $W$. As $\mathcal{F}_{b}^{\prime}$ is a simple foliation defined by $\pi$, we have $\mathcal{X}(W)=\ell\left(M, \mathcal{F}_{b}^{\prime}\right)$. whence the previous exact sequence becomes:

$$
0 \rightarrow \underline{\varepsilon}_{w} \rightarrow \ell\left(M, \mathcal{F}^{\prime}\right) \rightarrow \ell\left(M, \mathcal{F}_{b}^{\prime}\right) \rightarrow 0 .
$$

hence $\ell\left(M, \mathcal{F}_{b}^{\prime}\right) \subset \ell\left(M, \mathcal{F}^{\prime}\right)$.

This inducted us asking about the nature of relations existing between $\ell\left(M, \mathcal{F}^{\prime}\right)$ and $\ell\left(M, \mathcal{F}^{\prime \prime}\right)$ for any extension $\mathcal{F}^{\prime \prime}$ of riemannian foliation $\mathcal{F}^{\prime}$.

Proposition 4.2 Let $\mathcal{F}^{\prime \prime}$ an extension of a riemannian foliation $\mathcal{F}^{\prime}$ on a manifold $M$.

Then $\ell\left(M, \mathcal{F}^{\prime \prime}\right) \subset \ell\left(M, \mathcal{F}^{\prime}\right)$.

Proof. Let $X$ be a vector field $\mathcal{F}^{\prime \prime}$-transverse foliate, $h$ a $\mathcal{F}^{\prime}$-bundle-like metric of $\mathcal{F}^{\prime}$-transverse metric $h_{T}$ and $U$ a distinguished open set of $M$ both for $\mathcal{F}^{\prime}$ and $\mathcal{F}^{\prime \prime}$. There are three submersions $f^{\prime}: U \rightarrow \bar{U}$, $f^{\prime \prime}: U \rightarrow \overline{\bar{U}}$ and $\theta: \bar{U} \rightarrow \overline{\bar{U}}$ such that $\theta$ be the bond between $\left(U, \mathcal{F}^{\prime}\right)$ and $\left(U, \mathcal{F}^{\prime \prime}\right)$ and the restriction foliations $\left(U, \mathcal{F}^{\prime}\right)$ and $\left(U, \mathcal{F}^{\prime \prime}\right)$ are defined respectively by $f^{\prime}$ and $f^{\prime \prime}$.

The following diagram commutes:



The submersion $f^{\prime}$ is riemannian submersion which defines the riemannian foliation $\left(U, \mathcal{F}^{\prime}\right)$.

Now considering $P_{\left(x, \mathcal{F}^{\prime}\right)}^{U}$ and $P_{\left(x, \mathcal{F}^{\prime \prime}\right)}^{U}$ plaques respectively of $\mathcal{F}^{\prime}$ and $\mathcal{F}^{\prime \prime}$ in $U$ via $x \in U$.

Let $z \in P_{\left(x, \mathcal{F}^{\prime}\right)}^{U}$ and $\mathcal{F}_{\theta}$ the foliation in $\bar{U}$ defined by the submersion $\theta$. Let show that

$$
f_{* z}^{\prime}\left(\left(T_{z} \mathcal{F}^{\prime}\right)^{\perp} \cap\left(T_{z} \mathcal{F}^{\prime \prime}\right)\right)=T_{f^{\prime}(x)} \mathcal{F}_{\theta}
$$


We have $f^{\prime \prime}=\theta \circ f^{\prime}$. whence $f_{* z}^{\prime}\left(\left(T_{z} \mathcal{F}^{\prime}\right)^{\perp} \cap\left(T_{z} \mathcal{F}^{\prime \prime}\right)\right) \subset \operatorname{ker} \theta_{f^{\prime}(x)}$.

The vector spaces $f_{* z}^{\prime}\left(\left(T_{z} \mathcal{F}^{\prime}\right)^{\perp} \cap\left(T_{z} \mathcal{F}^{\prime \prime}\right)\right)$ and $\left(T_{z} \mathcal{F}^{\prime}\right)^{\perp} \cap\left(T_{z} \mathcal{F}^{\prime \prime}\right)$

have the same dimension because $f_{* z}^{\prime}:\left(T_{z} \mathcal{F}^{\prime}\right)^{\perp} \rightarrow T_{f^{\prime}(x)} \bar{U}$ is an isometry.

Therefore

$$
\begin{aligned}
& \operatorname{dim}\left(T_{z} \mathcal{F}^{\prime}\right)^{\perp} \cap\left(T_{z} \mathcal{F}^{\prime \prime}\right)=\operatorname{dim}\left(T_{z} \mathcal{F}^{\prime}\right)^{\perp}+\operatorname{dim} T_{z} \mathcal{F}^{\prime \prime}-\operatorname{dim}<\left(T_{z} \mathcal{F}^{\prime}\right)^{\perp} \cup T_{z} \mathcal{F}^{\prime \prime}> \\
&=\operatorname{dim} \mathcal{F}^{\prime \prime}-\operatorname{dim} \mathcal{F}^{\prime} \\
&=\operatorname{codim} \mathcal{F}^{\prime}-\operatorname{codim} \mathcal{F}^{\prime \prime} \\
&=\operatorname{dim} \operatorname{ker} \theta_{f^{\prime}(x)} . \\
& \text { so } f_{* z}^{\prime}\left(\left(T_{z} \mathcal{F}^{\prime}\right)^{\perp} \cap\left(T_{z} \mathcal{F}^{\prime \prime}\right)\right) \subset \operatorname{ker} \theta_{f^{\prime}(x)} \text { ensures that } \\
& f_{* z}^{\prime}\left(\left(T_{z} \mathcal{F}^{\prime}\right)^{\perp} \cap\left(T_{z} \mathcal{F}^{\prime \prime}\right)\right)=\operatorname{ker} \theta_{f^{\prime}(x)}=T_{f^{\prime}(x)} \mathcal{F}_{\theta} .
\end{aligned}
$$

That said, $X$ being $\mathcal{F}^{\prime \prime}$-transverse foliate, we have $f_{*}^{\prime \prime}(X)$ is a vector field on $\overline{\bar{U}}$.

It is noted $\overline{\bar{X}}$. More $X$ is valued in $\left(T \mathcal{F}^{\prime \prime}\right)^{\perp}$ because we identify the normal bundle $\mathcal{V}\left(\mathcal{F}^{\prime \prime}\right)$ of $\mathcal{F}^{\prime \prime}$ and the orthogonal bundle $\left(T \mathcal{F}^{\prime \prime}\right)^{\perp}$ of $T \mathcal{F}^{\prime \prime}$. Since

$$
\left(T \mathcal{F}^{\prime}\right)^{\perp}=\left(T \mathcal{F}^{\prime}\right)^{\perp} \cap\left(T \mathcal{F}^{\prime \prime}\right) \oplus\left(T \mathcal{F}^{\prime \prime}\right)^{\perp}
$$

and $f_{* z}^{\prime}:\left(T_{z} \mathcal{F}^{\prime}\right)^{\perp} \rightarrow T_{f^{\prime}(x)} \bar{U}$ is an isometry then for any $z \in P_{\left(x, \mathcal{F}^{\prime}\right)}^{U}$ and for any $Y_{z} \in\left(T_{z} \mathcal{F}^{\prime}\right)^{\perp} \cap\left(T_{z} \mathcal{F}^{\prime \prime}\right)$ we have

$$
0=h_{T}\left(Y_{z}, X_{z}\right)=\bar{h}_{T}\left(f_{* z}^{\prime}\left(Y_{z}\right), f_{* z}^{\prime}\left(X_{z}\right)\right)
$$

where $\bar{h}_{T}$ is the metric on $\bar{U}$ obtained by projection of $h_{T}$ on $\bar{U}$.

$\bar{h}_{T}\left(f_{* z}^{\prime}\left(Y_{z}\right), f_{* z}^{\prime}\left(X_{z}\right)\right)=0$ leads up that

$$
\begin{gathered}
f_{* z}^{\prime}\left(X_{z}\right) \in\left(f_{* z}^{\prime}\left(\left(T_{z} \mathcal{F}^{\prime}\right)^{\perp} \cap\left(T_{z} \mathcal{F}^{\prime \prime}\right)\right)\right)^{\perp} \text { for any } z \in P_{\left(x, \mathcal{F}^{\prime}\right)}^{U} \text {. Therefore } \\
f_{* z}^{\prime}\left(\left(T_{z} \mathcal{F}^{\prime}\right)^{\perp} \cap\left(T_{z} \mathcal{F}^{\prime \prime}\right)\right)=\operatorname{ker} \theta_{f^{\prime}(x)}=T_{f^{\prime}(x)} \mathcal{F}_{\theta}
\end{gathered}
$$

for every $z \in P_{\left(x, \mathcal{F}^{\prime}\right)}^{U}$, so

$$
f_{* z}^{\prime}\left(X_{z}\right) \in\left(T_{f^{\prime}(x)} \mathcal{F}_{\theta}\right)^{\perp}
$$

for every $z \in P_{\left(x, \mathcal{F}^{\prime}\right)}^{U}$.

We have $P_{\left(x, \mathcal{F}^{\prime}\right)}^{U} \subset P_{\left(x, \mathcal{F}^{\prime \prime}\right)}^{U}$. Whence for any $z, z^{\prime} \in P_{\left(x, \mathcal{F}^{\prime}\right)}^{U}$

$$
f_{* z}^{\prime \prime}\left(X_{z}\right)=f_{* z^{\prime}}^{\prime \prime}\left(X_{z^{\prime}}\right)=\overline{\bar{X}}_{f^{\prime \prime}(x)} .
$$


$f^{\prime \prime}=\theta \circ f^{\prime}$ leads up that

$$
\overline{\bar{X}}_{f^{\prime \prime}(x)}=f_{* z}^{\prime \prime}\left(X_{z}\right)=\theta_{* f^{\prime}(x)}\left(f_{* z}^{\prime}\left(X_{z}\right)\right)
$$

and

$$
\overline{\bar{X}}_{f^{\prime \prime}(x)}=f_{* z^{\prime}}^{\prime \prime}\left(X_{z^{\prime}}\right)=\theta_{* f^{\prime}(x)}\left(f_{* z^{\prime}}^{\prime}\left(X_{z^{\prime}}\right)\right)
$$

hence

$$
\theta_{* f^{\prime}(x)}\left(f_{* z}^{\prime}\left(X_{z}\right)\right)=\theta_{* f^{\prime}(x)}\left(f_{* z^{\prime}}^{\prime}\left(X_{z^{\prime}}\right)\right) .
$$

The fact that $\theta_{* f^{\prime}(x)}:\left(T_{f^{\prime}(x)} \mathcal{F}_{\theta}\right)^{\perp} \rightarrow T_{f^{\prime \prime}(x)} \overline{\bar{U}}$ be an isomorphism of vector spaces implies by using

$$
\begin{gathered}
\left(f_{* z}^{\prime}\left(X_{z}\right), f_{* z^{\prime}}^{\prime}\left(X_{z^{\prime}}\right)\right) \in\left(T_{f^{\prime}(x)} \mathcal{F}_{\theta}\right)^{\perp} \times\left(T_{f^{\prime}(x)} \mathcal{F}_{\theta}\right)^{\perp} \text { that } \\
f_{* z}^{\prime}\left(X_{z}\right)=f_{* z^{\prime}}^{\prime}\left(X_{z^{\prime}}\right)
\end{gathered}
$$

for any $z, z^{\prime} \in P_{\left(x, \mathcal{F}^{\prime}\right)}^{U}$.

It follows from that precedes the vector field $\mathcal{F}^{\prime \prime}$-transverse foliate $X$ is projeted according the plaques of $\mathcal{F}^{\prime}$ on the manifolds $\mathcal{F}^{\prime}$-local quotients of distinguished open sets for $\mathcal{F}^{\prime}$ and $\mathcal{F}^{\prime \prime}$. This shows that $X$ is a vector field $\mathcal{F}^{\prime}$-foliate.

Since $\left(T \mathcal{F}^{\prime \prime}\right)^{\perp} \subset\left(T \mathcal{F}^{\prime}\right)^{\perp}$ and $X$ is tangent to $\left(T \mathcal{F}^{\prime \prime}\right)^{\perp}$ then it shows that $X$ is tangent to $\left(T \mathcal{F}^{\prime}\right)^{\perp}$, hence $X$ is a vector field $\mathcal{F}^{\prime}$-foliate tangent to $\left(T \mathcal{F}^{\prime}\right)^{\perp}$. That shows that $X$ is a vector field $\mathcal{F}^{\prime}$-transverse foliate.

Properly $\ell\left(M, \mathcal{F}^{\prime \prime}\right) \subset \ell\left(M, \mathcal{F}^{\prime}\right)$.

Remark 4.3 We put $\bar{X}=f_{*}^{\prime}\left(X_{U}\right)$ where $X_{U}$ is a restriction to $U$ of the field $\mathcal{F}^{\prime \prime}$-transverse

foliate $X$. Note that $\bar{X}$ is tangent to $\left(T \mathcal{F}_{\theta}\right)^{\perp}$ and is projeted by $\theta$ on $\overline{\bar{X}}$.

It results that $\bar{X}$ is $\mathcal{F}_{\theta}$-transverse foliate.

Defitively, thanks to the commutative diagram

$$
\begin{aligned}
& \bar{U}
\end{aligned}
$$



that $f_{*}^{\prime}$ is a linear isomorphism between $\ell\left(U, \mathcal{F}^{\prime \prime}\right)$ and $\ell\left(\bar{U}, \mathcal{F}_{\theta}\right)$.

Using the above proposal and the theorems 2.4 and 3.1 we establish the following theorem which is the main result of this paper.

Theorem 4.4 Let $\mathcal{H}$ a Lie subalgebra of the Lie algebra $\mathcal{G}$ of a Lie-

foliation having dense leaves $\mathcal{F}$ on a compact manifold, $\omega$ a 1-form of Fedida defining

$\mathcal{F}$ and $\mathcal{F}_{\mathcal{H}}$ the extension of $\mathcal{F}$ corresponding to $\mathcal{H}$.

Then: 
i)

$$
\ell\left(M, \mathcal{F}_{\mathcal{H}}\right)=\bigcap_{h \in \mathcal{H}} \ell\left(M, \mathcal{F}_{<h>}\right)
$$

where $\mathcal{F}_{<h>}$ is the extension of $\mathcal{F}$ corresponding to the Lie subalgebra $<h>$ of $\mathcal{G}$ generated by $h$.

ii)

$$
\omega\left(\ell\left(M, \mathcal{F}_{\mathcal{H}}\right)\right) \subset \mathcal{H}^{\perp}
$$

where $\mathcal{H}^{\perp}$ is the ortho-complementary of $\mathcal{H}$ in $\mathcal{G}$ by the transverse metric associated to $\mathcal{F}$.

iii) For $h \in \mathcal{G}$,

$$
\omega\left(\ell\left(M, \mathcal{F}_{<h>}\right)\right)=\left\{u \in<h>^{\perp} /[u, h]=0\right\} .
$$

iv)

$$
\omega\left(\ell\left(M, \mathcal{F}_{\mathcal{H}}\right)\right)=\left\{u \in \mathcal{H}^{\perp} /[u, h]=0 \quad \text { for any } h \in \mathcal{H}\right\} .
$$

Proof. i) For every $h \in \mathcal{H}, \mathcal{F}_{<h>} \subset \mathcal{F}_{\mathcal{H}}$ and $\mathcal{F}_{<h>}$ is a riemannian foliation. Whence $\ell\left(M, \mathcal{F}_{\mathcal{H}}\right) \subset \ell\left(M, \mathcal{F}_{<h>}\right)$ for any $h \in \mathcal{H}$. This implies that $\ell\left(M, \mathcal{F}_{\mathcal{H}}\right) \subset \underset{h \in \mathcal{H}}{\cap} \ell\left(M, \mathcal{F}_{<h>}\right)$.

Let $Y \in \underset{h \in \mathcal{H}}{\cap} \ell\left(M, \mathcal{F}_{<h>}\right), Z$ a vector field tangent to $\mathcal{F}_{\mathcal{H}}$.

There is $X_{\mathcal{F}} \in \mathcal{X}(\mathcal{F}), X_{\mathcal{H}} \in \widetilde{\mathcal{H}}$ and $s \in \mathcal{A}^{1}(M)$ such that

$$
Z=X_{\mathcal{F}}+s \cdot X_{\mathcal{H}} .
$$

We have

$$
\begin{aligned}
{[Y, Z] } & =\left[Y, X_{\mathcal{F}}\right]+\left[Y, s \cdot X_{\mathcal{H}}\right] \\
& =\left[Y, X_{\mathcal{F}}\right]+Y(s) \cdot X_{\mathcal{H}}+s \cdot\left[Y, X_{\mathcal{H}}\right]
\end{aligned}
$$

However $Y$ is foliate for $\mathcal{F}_{<h>}$ so $Y$ is foliate for $\mathcal{F}$ because $\mathcal{F} \subset \mathcal{F}_{<h>}$ and $\mathcal{F}$ is riemannian. This shows that $\left[Y, X_{\mathcal{F}}\right]$ is tangent to $\mathcal{F} \subset \mathcal{F}_{\mathcal{H}}$. More $\omega\left(X_{\mathcal{H}}\right) \in \mathcal{H}$. whence $\left[Y, X_{\mathcal{H}}\right]$ is tangent to $\mathcal{F}_{\left\langle\omega\left(X_{\mathcal{H}}\right)\right\rangle} \subset \mathcal{F}_{\mathcal{H}}$. Given $Y(s) . X_{\mathcal{H}}$ tangent to $\mathcal{F}_{\mathcal{H}}$, It results from that precedes that $[Y, Z]$ is tangent to $\mathcal{F}_{\mathcal{H}}$. This means that $Y$ is $\mathcal{F}_{\mathcal{H}}$-transverse foliate. Hence $\cap_{h \in \mathcal{H}} \ell\left(M, \mathcal{F}_{<h>}\right) \subset \ell\left(M, \mathcal{F}_{\mathcal{H}}\right)$. Properly $\ell\left(M, \mathcal{F}_{\mathcal{H}}\right)=\bigcap_{h \in \mathcal{H}} \ell\left(M, \mathcal{F}_{<h>}\right)$.

ii) Noted that for every $X \in \ell(M, \mathcal{F})$ and for every $x \in M$ we have $\omega_{x}\left(X_{x}\right)=\omega(X)$ because the Lie-foliation $\mathcal{F}$ is with dense leaves. Let $U$ a local open of trivialization of universal covering $\widetilde{M}$ of $M$ distinguished both for $\mathcal{F}$ and $\mathcal{F}_{\mathcal{H}}$.

There is (cf.remark 2.5) a riemannian submersion $f$ defining $\mathcal{F}$ on $U$ and checking

$$
\omega_{x}\left(X_{x}\right)=\left(L_{f(x) *}\right)^{-1} \circ f_{* x}\left(X_{x}\right)
$$

for every $X_{x} \in T_{x} U$. 
That said, we have

$$
\begin{aligned}
\omega\left(\ell\left(M, \mathcal{F}_{\mathcal{H}}\right)\right) & =\omega_{x}\left(\ell\left(M, \mathcal{F}_{\mathcal{H}}\right)\right) \\
& =\left(L_{f(x) *}\right)^{-1} \circ f_{* x}\left(\ell\left(M, \mathcal{F}_{\mathcal{H}}\right)\right) .
\end{aligned}
$$

But according to the remark 4.2

$$
f_{* x}\left(\ell\left(M, \mathcal{F}_{\mathcal{H}}\right)\right) \subset\left(\operatorname{Ker} \theta_{f(x)}\right)^{\perp}=\left(L_{f(x) *}(\mathcal{H})\right)^{\perp}
$$

then $\omega\left(\ell\left(M, \mathcal{F}_{\mathcal{H}}\right)\right) \subset\left(L_{f(x) *}\right)^{-1}\left(L_{f(x) *}(\mathcal{H})\right)^{\perp}$.

we also have $\left(L_{f(x) *}\right)^{-1}\left(L_{f(x) *}(\mathcal{H})\right)^{\perp}=\mathcal{H}^{\perp}$ because $L_{f(x) *}$ is an isometry. It results from the foregoing that $\omega\left(\ell\left(M, \mathcal{F}_{\mathcal{H}}\right)\right) \subset \mathcal{H}^{\perp}$.

iii) Let $v \in \omega\left(\ell\left(M, \mathcal{F}_{<h>}\right)\right)$ and $\widetilde{h}$ field $\mathcal{F}$-transverse foliate associated to $h$.

Since $v \in \omega\left(\ell\left(M, \mathcal{F}_{<h>}\right)\right)$ then there is $\widetilde{v} \in \ell\left(M, \mathcal{F}_{<h>}\right)$ such that $\omega(\widetilde{v})=v$.

We have $[\widetilde{v}, \widetilde{h}]$ tangent to $\mathcal{F}_{<h>}$ as $\widetilde{h}$ is tangent to $\mathcal{F}_{<h>}$ and $\widetilde{v} \in \ell\left(M, \mathcal{F}_{<h>}\right)$.

Accordingly

$$
\omega([\widetilde{v}, \widetilde{h}]) \in \omega\left(T \mathcal{F}_{<h>}\right)=<h>
$$

where $\langle h\rangle$ is the Lie subalgebra generated by $h$.

More, the Lie-foliation $\mathcal{F}$ being with dense leaves, we have

$$
\omega([\widetilde{v}, \widetilde{h}])=[\omega(\widetilde{v}), \omega(\widetilde{h})]=[v, h] .
$$

It results from foregoing that $[v, h] \in\langle h\rangle$. This implies that $[v, h]=0$ as $[v, h]$ is orthogonal to vectors $v$ and $h$.

hence the fact that $\omega\left(\ell\left(M, \mathcal{F}_{<h>}\right)\right) \subset<h>^{\perp}$ ensures that $v \in\left\{u \in<h>^{\perp} /[u, h]=0\right\}$.

Let $b \in\left\{u \in<h>^{\perp} /[u, h]=0\right\}, \widetilde{b}$ the field $\mathcal{F}$-transverse foliate associated to $b$ and $X$ a vector field tangent to $\mathcal{F}_{\langle h\rangle}$. There is $X_{\mathcal{F}} \in \mathcal{X}(\mathcal{F})$ and $s \in \mathcal{A}^{1}(M)$ such that

$$
X=X_{\mathcal{F}}+s \cdot \widetilde{h}
$$

whence

$$
\begin{aligned}
{[\widetilde{b}, X] } & =\left[\widetilde{b}, X_{\mathcal{F}}\right]+[\widetilde{b}, s \cdot \widetilde{h}] \\
& =\left[\widetilde{b}, X_{\mathcal{F}}\right]+\widetilde{b}(s) \cdot \widetilde{h}+s \cdot[\widetilde{b}, \widetilde{h}]
\end{aligned}
$$

Hence

$$
\begin{aligned}
\omega([\widetilde{b}, X]) & =\omega\left(\left[\widetilde{b}, X_{\mathcal{F}}\right]\right)+\omega(\widetilde{b}(s) \cdot \widetilde{h})+\omega(s \cdot[\widetilde{b}, \widetilde{h}]) \\
& =\widetilde{b}(s) \cdot \omega(\widetilde{h})+s \cdot \omega([\widetilde{b}, \widetilde{h}]) \\
& =\widetilde{b}(s) \cdot h .
\end{aligned}
$$


$\omega([\widetilde{b}, X])=\widetilde{b}(s) . h$ shows that $[\widetilde{b}, X]$ is tangent to $\mathcal{F}_{<h>}$

(cf. remark 2.5). Accordingly $\widetilde{b}$ is foliate for $\mathcal{F}_{\langle h\rangle}$.

Let $U$ a local open of trivialization of universal covering $\widetilde{M}$ of $M$

distinguished both for $\mathcal{F}$ and $\mathcal{F}_{\langle h\rangle}, h_{T}$ the bundle-like metric $\mathcal{F}$-transverse on $U$ and $\bar{h}_{T}$ the left-invariant metric on $\bar{U}$ associated to $h_{T}$.

There is (cf.remark 2.5) a riemannian submersion $f$ defining $\mathcal{F}$ on $U$ and verifying

$$
\omega_{x}\left(X_{x}\right)=\left(L_{f(x) *}\right)^{-1} \circ f_{* x}\left(X_{x}\right)
$$

For every $X_{x} \in T_{x} U$.

For every $x \in U$ we have:

$$
\begin{aligned}
h_{T}\left(\widetilde{b}_{x}, \widetilde{h}_{x}\right) & =\bar{h}_{T}\left(f_{* x}\left(\widetilde{b}_{x}\right), f_{* x}\left(\widetilde{h}_{x}\right)\right) \\
& =\bar{h}_{T}\left(\left(L_{f(x) *}\right)^{-1} \circ f_{* x}\left(\widetilde{b}_{x}\right),\left(L_{f(x) *}\right)^{-1} \circ f_{* x}\left(\widetilde{h}_{x}\right)\right) \\
& =\bar{h}_{T}\left(\omega_{x}\left(\widetilde{b}_{x}\right), \omega_{x}\left(\widetilde{h}_{x}\right)\right) \\
& =\bar{h}_{T}(b, h) \\
& =0
\end{aligned}
$$

hence the vector field $\widetilde{b}$ foliate for $\mathcal{F}_{<h>}$ is $\mathcal{F}_{<h>}$-transverse foliate . i.e. $\widetilde{b} \in \ell\left(M, \mathcal{F}_{<h>}\right)$. This implies that $b \in \omega\left(\ell\left(M, \mathcal{F}_{<h>}\right)\right)$.

It results from the above that

$$
\omega\left(\ell\left(M, \mathcal{F}_{<h>}\right)\right)=\left\{u \in<h>^{\perp} /[u, h]=0\right\} .
$$

iv) It is easy to see that

$$
\omega\left(\underset{h \in \mathcal{H}}{\cap} \ell\left(M, \mathcal{F}_{<h>}\right)\right) \subset \underset{h \in \mathcal{H}}{\cap} \omega\left(\ell\left(M, \mathcal{F}_{<h>}\right)\right) .
$$

Now, let us prove that

$$
\bigcap_{h \in \mathcal{H}} \omega\left(\ell\left(M, \mathcal{F}_{<h>}\right)\right) \subset \omega\left(\underset{h \in \mathcal{H}}{\cap} \ell\left(M, \mathcal{F}_{<h>}\right)\right) .
$$

Let $u \in \underset{h \in \mathcal{H}}{\cap} \omega\left(\ell\left(M, \mathcal{F}_{<h>}\right)\right)$.

For every $h \in \mathcal{H}$ there is $\widetilde{u}_{h} \in \ell\left(M, \mathcal{F}_{<h>}\right)$ such that $\omega\left(\widetilde{u}_{h}\right)=u$.

Since $\mathcal{F}_{<h>}$ is an extension of foliation $\mathcal{F}$ riemannian then $\widetilde{u}_{h} \in \ell(M, \mathcal{F})$. The foliation $\mathcal{F}$ is a Lie-foliation with dense leaves, whence $\omega$ is an linear isomorphism between $\ell(M, \mathcal{F})$ and $\mathcal{G}$. Hence $\omega\left(\widetilde{u}_{h}\right)=u$ for any

$h \in \mathcal{H}$ leads up that for any $h_{0} \in \mathcal{H}$ and any $h_{1} \in \mathcal{H}$ we have $\widetilde{u}_{h_{0}}=\widetilde{u}_{h_{1}}$. We can state $\widetilde{u}_{h}=\widetilde{u}$ for all $h \in \mathcal{H}$.

It is easy to see that $\widetilde{u} \in \underset{h \in \mathcal{H}}{\cap} \ell\left(M, \mathcal{F}_{<h>}\right)$. This implies that $u \in \omega\left(\underset{h \in \mathcal{H}}{\cap} \ell\left(M, \mathcal{F}_{<h>}\right)\right)$. 
It results from the above that

$$
\bigcap_{h \in \mathcal{H}} \omega\left(\ell\left(M, \mathcal{F}_{<h>}\right)\right) \subset \omega\left(\bigcap_{h \in \mathcal{H}} \ell\left(M, \mathcal{F}_{<h>}\right)\right) .
$$

Properly we have

$$
\bigcap_{h \in \mathcal{H}} \omega\left(\ell\left(M, \mathcal{F}_{<h>}\right)\right)=\omega\left(\bigcap_{h \in \mathcal{H}} \ell\left(M, \mathcal{F}_{<h>}\right)\right) .
$$

By

$$
\ell\left(M, \mathcal{F}_{\mathcal{H}}\right)=\bigcap_{h \in \mathcal{H}} \ell\left(M, \mathcal{F}_{<h>}\right)
$$

and

$$
\omega\left(\ell\left(M, \mathcal{F}_{<h>}\right)\right)=\left\{u \in<h>^{\perp} /[u, h]=0\right\}
$$

we obtain

$$
\begin{aligned}
\omega\left(\ell\left(M, \mathcal{F}_{\mathcal{H}}\right)\right) & =\omega\left(\underset{h \in \mathcal{H}}{\cap} \ell\left(M, \mathcal{F}_{<h>}\right)\right) \\
& =\underset{h \in \mathcal{H}}{\cap} \omega\left(\ell\left(M, \mathcal{F}_{<h>}\right)\right) \\
& =\underset{h \in \mathcal{H}}{\bigcap}\left\{u \in<h>^{\perp} /[u, h]=0\right\} \\
& =\left\{u \in \mathcal{H}^{\perp} /[u, h]=0 \text { for every } h \in \mathcal{H}\right\} .
\end{aligned}
$$

We note using vi) of remark 2.5 that $\omega$ realizes a Lie algebra isomorphism between $\ell\left(M, \mathcal{F}_{\mathcal{H}}\right)$ and $\left\{u \in \mathcal{H}^{\perp} /[u, h]=0\right.$ for every $\left.h \in \mathcal{H}\right\}$.

We also note that we can show that $\left\{u \in \mathcal{H}^{\perp} /[u, h]=0\right.$ for all $\left.h \in \mathcal{H}\right\}$

is a Lie subalgebra without using $\omega$. As an illustration,

let's observe two vectors $u$ and $v$ of $\left\{u \in \mathcal{H}^{\perp} /[u, h]=0\right.$ for every $\left.h \in \mathcal{H}\right\}$.

We have for $k \in \mathcal{H}$,

$$
\begin{aligned}
0 & =[[u, v], k]+[[v, k], u]+[[k, u], v] \\
& =[[u, v], k]+[0, u]+[0, v] \\
& =[[u, v], k]
\end{aligned}
$$

Moreover if $\bar{h}_{T}$ is a left-invariant metric on the Lie group $G$ we have

$$
\begin{aligned}
0 & =\widetilde{u} \cdot \bar{h}_{T}(\widetilde{v}, \widetilde{k}) \\
& =\bar{h}_{T}([\widetilde{u}, \widetilde{v}], \widetilde{k})+\bar{h}_{T}(\widetilde{v},[\widetilde{u}, \widetilde{k}]) \\
& =\bar{h}_{T}\left([\widetilde{u}, \widetilde{v}], k^{g}\right)+\bar{h}_{T}(\widetilde{v}, 0) \\
& =\bar{h}_{T}([\widetilde{u}, \widetilde{v}], \widetilde{k}) \\
& =\bar{h}_{T}([u, v], k)
\end{aligned}
$$


because the foliated transverse fieldes $\widetilde{u}, \widetilde{v}$ and $\widetilde{k}$ associated respectively to $u$,

$v$ and $k$ are Killing vectors fields.

$[[u, v], k]=0$ and $\bar{h}_{T}([u, v], k)=0$ for every $k \in \mathcal{H}$ ensures that $[u, v]$

is a vector of $\left\{u \in \mathcal{H}^{\perp} /[u, h]=0\right.$ fo every $\left.h \in \mathcal{H}\right\}$.

Noted at the end that if $\widetilde{\mathcal{H}}$ is the Lie algebra of vector fields $\mathcal{F}$-transverse foliate obtained from $\mathcal{H}$ then

$$
\omega\left(\ell\left(M, \mathcal{F}_{\mathcal{H}}\right)\right)=\left\{u \in \mathcal{H}^{\perp} /[u, h]=0 \text { for every } h \in \mathcal{H}\right\}
$$

that shows $X \in \ell\left(M, \mathcal{F}_{\mathcal{H}}\right), Y \in \widetilde{\mathcal{H}}$ and the vector field $\mathcal{F}$-transverse foliate $[X, Y]$ is zero. Indeed

$$
\omega([X, Y])=[\omega(X), \omega(Y)]=0
$$

because $\omega(X) \in\left\{u \in \mathcal{H}^{\perp} /[u, h]=0\right.$ for every $\left.h \in \mathcal{H}\right\}$ and $\omega(Y) \in \mathcal{H}$.

The respective projection $\bar{X}$ and $\bar{Y}$ of $X$ and $Y$ on a local quotient manifold $\bar{U}$ for $\mathcal{F}$ are left-invariant fields. whence for every $g \in \bar{U}$ we have

$$
[\bar{X}, \bar{Y}]_{g}=L_{g *}([\omega(X), \omega(Y)])=0 .
$$

Hence $[\bar{X}, \bar{Y}]=0$. This shows that for every $x \in M$

$$
\left(\varphi_{t}^{Y}\right)_{* x}\left(X_{x}\right)=X_{\varphi_{t}^{Y}(x)}
$$

where $\varphi_{t}^{Y}$ is the flow of $Y$.

Corollary 4.5 Let $\mathcal{G}$ the Lie algebra of a Lie-foliation having dense leaves $\mathcal{F}$ on a compact

manifold.

We have:

i) If $\mathcal{H}$ is an ideal of $\mathcal{G}$ then the ortho-complémentary $\mathcal{H}^{\perp}$ of $\mathcal{H}$ in

$\mathcal{G}$ is also an ideal,

ii) If $\mathcal{F}$ has an extension $\mathcal{F}^{\prime}$ of Lie then $\mathcal{F}$ has also an other extension $\mathcal{F}^{\prime \prime}$ of Lie such that $(T \mathcal{F})^{\perp} \cap\left(T \mathcal{F}^{\prime}\right)$ be orthogonal to $(T \mathcal{F})^{\perp} \cap\left(T \mathcal{F}^{\prime \prime}\right)$.

Proof. Let $\omega$ be a 1 -form of fédida defining the Lie-foliation having dense leaves $\mathcal{F}$.

i) Supposed that $\mathcal{H}$ is an ideal of $\mathcal{G}$.

From theorem 2.4, the extension $\mathcal{F}_{\mathcal{H}}$ of $\mathcal{F}$ corresponding to $\mathcal{H}$ is a Lie-foliation. Whence ([3], [4])

$$
\operatorname{dim} \omega\left(\ell\left(M, \mathcal{F}_{\mathcal{H}}\right)\right)=\operatorname{dim}\left(\ell\left(M, \mathcal{F}_{\mathcal{H}}\right)\right)=\operatorname{dim}\left(\frac{\mathcal{G}}{\mathcal{H}}\right)=\operatorname{dim}\left(\mathcal{H}^{\perp}\right) .
$$

But $\omega\left(\ell\left(M, \mathcal{F}_{\mathcal{H}}\right)\right) \subset \mathcal{H}^{\perp}$ then $\omega\left(\ell\left(M, \mathcal{F}_{\mathcal{H}}\right)\right)=\mathcal{H}^{\perp}$. This shows that $\mathcal{H}^{\perp}$ is a Lie subalgebra of $\mathcal{G}$. 
Since $\omega\left(\ell\left(M, \mathcal{F}_{\mathcal{H}}\right)\right)=\mathcal{H}^{\perp}$ then from the theorem 4.3 we have $[h, u]=0$ for every $h \in \mathcal{H}$ and all $u \in \mathcal{H}^{\perp}$.

Let $v \in \mathcal{G}$,

there is $v_{\mathcal{H}} \in \mathcal{H}$ and $v_{\mathcal{H}^{\perp}} \in \mathcal{H}^{\perp}$ such that $v=v_{\mathcal{H}}+v_{\mathcal{H}^{\perp}}$.

Whence for every $u \in \mathcal{H}$,

$$
[v, u]=\left[v_{\mathcal{H}}, u\right]+\left[v_{\mathcal{H}^{\perp}}, u\right]=\left[v_{\mathcal{H}}, u\right] .
$$

It results of the fact that $\mathcal{H}^{\perp}$ be a Lie subalgebra that $[v, u] \in \mathcal{H}^{\perp}$ for every $u \in \mathcal{H}$ and all $v \in \mathcal{G}$. i.e. $\mathcal{H}^{\perp}$ is an ideal of $\mathcal{G}$.

ii) Supposed that $\mathcal{F}$ has an extension $\mathcal{F}^{\prime}$ of Lie.

From the theorem 2.4there is an ideal $\mathcal{H}$ of $\mathcal{G}$ corresponding to $\mathcal{F}^{\prime}$.

It results from i) that $\mathcal{H}^{\perp}$ is also an ideal of $\mathcal{G}$.

Let $\mathcal{F}^{\prime \prime}$ be the extension of $\mathcal{F}$ corresponding to $\mathcal{H}^{\perp}$.

we have

$$
\omega\left((T \mathcal{F})^{\perp} \cap\left(T \mathcal{F}^{\prime}\right)\right)=\mathcal{H} \text { and } \omega\left((T \mathcal{F})^{\perp} \cap\left(T \mathcal{F}^{\prime \prime}\right)\right)=\mathcal{H}^{\perp} .
$$

According to remark 2.5 it is taken $\omega$ which can be localy the darboux's differential of riemannian submersion. This implies that $(T \mathcal{F})^{\perp} \cap\left(T \mathcal{F}^{\prime}\right)$ is orthogonal to $(T \mathcal{F})^{\perp} \cap\left(T \mathcal{F}^{\prime \prime}\right)$.

\section{References}

[1] R.Almeida et P.Molino, 1986. "Flot riemanniens sur les 4-variétés compactes" Tôhoku Mathematical Journal, The Second Series, Vol. 38, no. 2, pp. 313-326.

[2] Y.Carrière, "Flots Riemanniens. In Structures transverses des feuilletages", Astérisque, 116 (1984), 31-52.

[3] C.Dadi, 2008. "Sur les extensions des feuilletages". Thèse unique, Université de Cocody, Abidjan.

[4] C.Dadi and H.Diallo, 2007. "Extension d'un feuilletage de Lie minimal d'une variété compacte". Afrika Matematika, Série 3, volume 18 pp 34-45.

[5] E.Fédida, 1974. "Sur l'existence des Feuilletages de Lie". CRAS de Paris, $278,835-837$.

[6] C.Godbillon, 1985. "Feuilletage; Etude géométriques I". Publ, IRMA, Strasbourg .

[7] P.Molino, 1988. "Riemannian foliations". Birkhäuser.

[8] T.Masson, 2001. "Géométrie différentielle, groupes et algèbres de Lie, fibrés et connexions", laboratoire de Physique Théorique, Université Paris XI, Bâtiment 210, 91405 Orsay Cedex France. 
[9] C.Dadi and A.Codjia, 2016 "Riemannian foliation with dense leaves on a compact manifold". Internatonal Journal of Mathematics and Computeur science 11 no 2 151- 172 . 